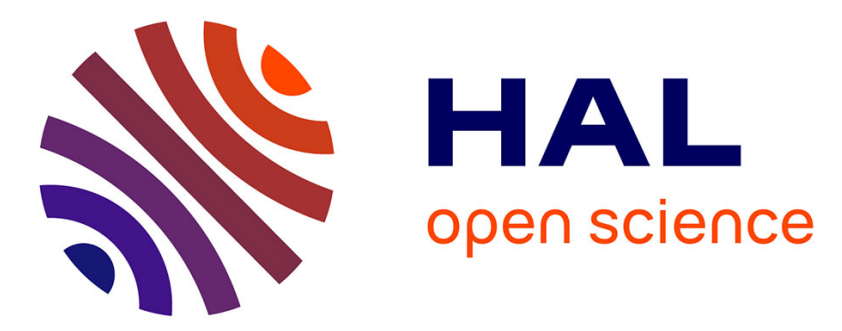

\title{
Exploiting distance technology to foster experimental design as a neglected learning objective in labwork in chemistry
}

Cedric d'Ham, Erica de Vries, Isabelle Girault, Patricia Marzin-Janvier

\section{- To cite this version:}

Cedric d'Ham, Erica de Vries, Isabelle Girault, Patricia Marzin-Janvier. Exploiting distance technology to foster experimental design as a neglected learning objective in labwork in chemistry. Journal of Science Education and Technology, 2004, 13(4), pp.425-434. hal-00190183

\section{HAL Id: hal-00190183 \\ https://telearn.archives-ouvertes.fr/hal-00190183}

Submitted on 23 Nov 2007

HAL is a multi-disciplinary open access archive for the deposit and dissemination of scientific research documents, whether they are published or not. The documents may come from teaching and research institutions in France or abroad, or from public or private research centers.
L'archive ouverte pluridisciplinaire HAL, est destinée au dépôt et à la diffusion de documents scientifiques de niveau recherche, publiés ou non, émanant des établissements d'enseignement et de recherche français ou étrangers, des laboratoires publics ou privés. 


\title{
Exploiting distance technology to foster experimental design as a neglected learning objective in labwork in chemistry
}

\author{
Cédric d'Ham ${ }^{1}$, Erica de Vries ${ }^{2}$, Isabelle Girault ${ }^{1} \&$ Patricia Marzin $^{1}$ \\ ${ }^{1}$ Laboratoire Interdisciplinaire de Didactique des Sciences Expérimentales et des Technologies - \\ Université Joseph Fourier de Grenoble, BP 53, 38041 Grenoble cedex 9, France \\ Tel: +33 4765141 86, Fax: +33 4765148 35, cdham@ujf-grenoble.fr \\ ${ }^{2}$ Laboratoire des Sciences de l'Education - Université Pierre-Mèndes-France de Grenoble
}

The original publication is available at www.springerlink.com

\begin{abstract}
This article deals with the design process of a remote laboratory for labwork in chemistry. In particular, it focuses on the mutual dependency of theoretical conjectures about learning in the experimental sciences and technological opportunities in creating learning environments. The design process involves a detailed analysis of the expert task and knowledge, e.g., spectrophotometry as a method for the determination of the concentration of a compound in a solution. In so doing, modifications in transposing tasks and knowledge to the learning situation can be monitored. The remote laboratory is described, as well as the specific features that alter the degree of fidelity of the learning situation in comparison to the expert one. It is conjectured that these alterations might represent actual benefits for learning.
\end{abstract}

\section{Introduction}

Nowadays, numerous learning environments are proposed and developed that involve some application of Information and Communication Technology (ICT). The design of such computersupported learning environments can be characterized as either technology-driven or theory-driven. On the one hand, technological progress influences the design of learning environments through the availability of new tools and techniques. On the other hand, advances in learning theory show the way to new roles and possible uses of technology for learning. Both technology and theory-driven development of learning environments tend to result in some adaptation of the expert domain knowledge and tasks that are the goal of the teaching. The general process of turning domain knowledge into an object of teaching has been called didactical transposition (Chevallard 1985), and more specifically, it has been coined computational transposition (Balacheff 1996) when it comes to 
designing learning environments involving ICT. Computational transposition is the process which leads to the specification and the implementation of the knowledge model; i.e. adapting domain knowledge and tasks requires effort in order to fit the constraints of symbolic representation and computation imposed by the computer. On the other hand, the computer also allows new ways of manipulating representations, and these opportunities, in combination with knowledge from studies of expert tasks, should inform the design of new learning environments (see also Kozma et al. 2000).

The current article focuses on this mutual dependency of theoretical conjectures and technological opportunities in the particular case of the design of a remote laboratory for labwork in chemistry.

\section{ICT for labwork in the experimental sciences}

The literature shows a proliferation of computer programs for learning in the experimental sciences. Theories, concepts, laws, and phenomena in chemistry and physics can be presented and explained, but also documented, illustrated, modelled, depicted, etc. using a variety of computer programs and displays. A number of different theoretical frameworks on learning constitute the foundations of such environments (see De Vries 2003, for an overview). More specifically, regarding labwork in the experimental sciences, such environments include systems providing access to animations, simulations, pre-recorded experimental data and remote experimental devices. Trgalová (2003) proposes a categorization on the basis of two criteria, i.e. the nature of experimental data and the degree of user control. Animations and simulations deal with simulated data, no real experiments are carried out, but the results of fictitious experiments are presented to the students. In the case of simulations, the learner can vary the value of input variables and observe the outcomes in terms of output variables. This type of computer programs is used to encourage discovery learning, i.e. students themselves discover the laws of a domain by carrying out virtual experiments on a model of that domain (de Jong and van Joolingen 1998). Animations do not allow such a high degree of user control, they merely present dynamic illustrations of laws and/or phenomena. Pre-recorded experimental data and remote experimental devices deal with data obtained from real experiments. They differ in the time of data collection, the former data are pre-recorded, whereas the latter are realtime collected. In addition, real-time collected data differ in the degree of user control (Trgalová 2003): none (remote sensing devices), limited (simple remote operation devices) or full (remote laboratory). The appropriateness of each of these technologies for learning should be gauged in reference to pedagogical objectives.

\section{Technology-induced changes in learning contexts, tasks and knowledge}

Whereas remote laboratories dedicated to chemistry experiments may provide almost full student control via the Internet, such as for example in Senese et al. (2000) or in Cooper et al. (2002), students are often only allowed to control the parameters of a pre-designed experiment. Students in the latter situation are not expected to actually design the experiment, although it is in fact an essential subtask of a chemist to consider several alternative experimental settings. Moreover, the use of ICT might change the nature of labwork in chemistry in a number of other non-trivial ways. For example, tasks may either be formalized or remain implicit, manipulations can be taken over by a robot, students may or may not decide on the material used, security norms may be imposed by the system, etc. Still other aspects involve the educational setting, for example an artificial tutor might take over the role of the teacher, a robot might manipulate and carry out the experiment instead of the students themselves, the pedagogical strategy of the artificial tutor can be programmed to provide help or not. In order to control such technology-driven changes, a more systematic approach is needed that first analyzes 
domain tasks and knowledge, and then studies how they change in the process of implementing components of the learning environment. The adaptation of domain tasks and knowledge can then be monitored so as to produce presumably positive changes instead of mere arbitrary or even negative changes. One of our goals in designing the remote laboratory is therefore to coordinate and monitor modifications and adaptations of the expert domain knowledge and tasks.

The second section presents a review of the literature showing how the subtask of experimental design is often neglected in teaching practices to the advantage of other subtasks, such as carrying out an experiment, analysing data, etc. In contrast, our approach entails proposing a starting question and making the learner focus on the design of the experimental protocol that allows for data collection and finding an answer. The activity of designing a protocol is likely to produce a confrontation between theory, problem and experiment. This confrontation is an important way to encourage students to construct meaning and to acquire knowledge that can be reinvested in practical work. The third section then presents the design of Educaffix.net, a system that exploits the features of a remote laboratory for focusing on experimental design. This includes the systematic analysis of domain tasks and concepts in labwork in chemistry that guided the design process. A specific set of domain concepts is presented: the determination of the concentration of a solution with the help of photometry. In the fourth section, specific technology-based features will be highlighted in a comparison with the expert situation. The article concludes with perspectives for future research.

\section{Labwork in science education}

Practical work in a laboratory is essential for the learner in experimental sciences because some specific learning objectives can only be met in this kind of situation. According to Becu-Robinault (1997), there are experiments for seeing a phenomenon, experiments for introducing a concept, and experiments for verifying a scientific law.

\section{Multiple pedagogical objectives}

Several studies classified the learning objectives that teachers can associate with student labwork. Table 1 shows a number of possible learning objectives for labwork (Tiberghien et al. 2001).

Table 1. Learning objectives for labwork sessions (Tiberghien, et al., 2001)

1 - Identify objects and phenomena and become familiar with them

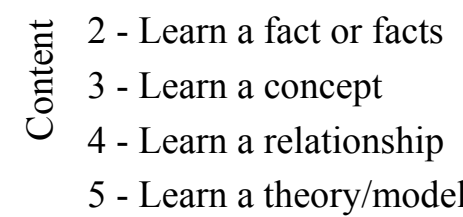

6 - Learn how to use a standard laboratory instrument or piece of apparatus

7 - Learn how to carry out a standard procedure

过 8 - Learn how to plan an investigation in order to address a specific question or problem

. 9 - Learn how to process data

10 - Learn how to use data to support a conclusion

11 - Learn how to communicate the results of their work 
For the purposes of the present article, two additional observations are necessary: the nature of objectives in the process category and the interdependency of objectives 7 and 8 . Regarding the nature of the objectives, it can be noticed that process objectives are of two categories, some have a manual nature. In particular, objective 6 in Table 1 concerns the gestures that an expert chemist carries out in order to do experiments, whereas the objectives 7 through 11 on the other hand are largely methodological and concern a number of steps and their order for the activities mentioned. Regarding the objectives 7 and 8, planning an investigation (objective 8) additionally involves knowing how to apply a standard procedure (objective 7) or even how to design one oneself. The latter would involve learning the principles of a standard experimental procedure, which in itself might be more content like.

Among the process objectives, Tiberghien et al. (2001) showed that "Learn how to use a standard laboratory instrument" is very common in chemistry in the countries studied, whereas "Learn how to plan an investigation to address a specific question or problem" is infrequent in chemistry. Furthermore, labwork sessions are usually found to be sequenced in four phases corresponding to the following tasks:

- Task 1: prepare / design the experiment

- Task 2: carry out the experiment (genuine practical labwork)

- Task 3: use the results to solve the scientific question

- Task 4: communicate the results

Whereas tasks 2 and 3 are constitutive of a labwork session and cannot be totally removed, task 4 is a general non-specific task. Thus, the main variables that can change the context of a labwork session are related to Task 1 . The learning objectives shown in Table 1 are often preferentially present in one selected task.

\section{Impact of teachers' view on science}

The teacher, depending on his or her own personal view of experimental sciences, can emphasize some selected tasks, while minimizing others. In fact, teachers may adopt one of a variety of different points of view of science. Novak (1988), Orlandi (1991) and Desautel et al. (1993) studied these novice and expert teachers' images of science. They found that teachers hold a view according to which a scientific method is a linear and rational process in the pursuit of the truth. They also showed that a majority of teachers hold essentially positivist views of the nature of knowledge and knowledge production. These views are likely to influence teaching about science and the objectives that can be met in teacher-organised labwork sessions.

\section{Focusing on experimental design}

Sere and Beney (1997) described and analyzed intellectual activities of students in labwork activities; a majority of students were shown to call upon other references than scientific theories to conduct their experimental activities. Some of these references, notably those that differ from the way in which science operates, may contribute to the construction of misconceptions about science. The general objective of learning in the experimental sciences, as proposed by Darley (1996) for example, is the ability for the students to define procedures for testing hypotheses and/or models initially proposed during a problem analysis phase. Thus, students should be involved in situations where they are novice 
researchers. Others (Arce and Betancourt 1997, Séré 2002) also emphasized the importance of the task of experimental design in a learning context.

Since experimental design task is complex in itself, errors are very likely to occur especially in beginners. Consequently, students are hardly ever allowed to design their own experiment and then carry it out with real chemicals and laboratory apparatus. The study by Tiberghien et al. (2001) showed that the most relevant learning objective in labwork (objective 8: to learn how to plan an investigation in order to address a specific question or problem) was in fact the least frequent process objective, it was mentioned in less than $15 \%$ of the sample studied. One of the reasons could be that errors in experimental design highly influence the actual labwork tasks 2 and 3. Errors in the experimental procedure might create dangerous situations during the experiment itself (Tasks 2: doing an experiment). Moreover, even if the execution of the experiment does not give rise to problems in itself, problems are likely to occur when interpreting the results, e.g., in case of inconclusive data (Task 3: interpreting the results). Therefore, it seems reasonable in a number of situations, to favour Tasks 2 and 3 at the expense of experimental design (Task 1). Moreover, correct performance of tasks 2 and 3 are considered of crucial importance in learning to manipulate (manual component) and in conceptualising about the domain (content knowledge component).

\section{A psychological task analysis of experimental design}

From an epistemological point of view, the task of designing an experiment in order to answer a research question is an important skill. Experimental design is the main procedure for accumulating evidence in traditional science domains (physics, chemistry, biology) but also in the social sciences, such as in psychology. High quality experimental design warrants validity and reliability of the results. Experimental design as an expert task in the social sciences has been studied by Schraagen (1993). His theoretical task analysis shows two important sub-goals, 1) answer the research question and 2) control sources of variance (Schraagen, 1993). These correspond to the main concerns to be monitored during task performance. Although the subgoals themselves are independent of the specific domain of investigation, the input in terms of content heavily relies on domain knowledge, e.g., what kinds of phenomena may cause error variance in a particular domain. For example, in designing an experiment on people's preferences regarding the flavour of different beverages, the administration procedure for tasting several beverages one after the other has to be designed so as to minimise carry-over effects. Equivalent problems can be found in other domains, e.g. in a chemistry lab, reusing flasks poses problems of polluted fluids. Different solutions will be adopted in the different domains. In the psychology domain, a solution would involve varying the order in which the beverages are administered and by giving something to eat in between beverages; in the chemistry domain, a solution would involve rinsing flasks with the right liquid before (re)using them. The construction of these different solutions to an experimental design problem thus is highly affected by domain knowledge.

Experimental design also shows commonalities with design processes in other domains (architecture, mechanical design, etc.). These commonalities can be illustrated by referring to the three defining characteristics of design processes (e.g. de Vries 1994). First, design involves the construction of something new as opposed to calculating a solution with the help of an algorithm or copying an old solution to the problem. In experimental design, existing paradigms, procedures or protocols can be reused, but they need to be adapted to the specificities of each new situation. Second, design involves constructing a representation or plan of something before it is manufactured, e.g. the drawing of a house. In experimental design, it is the procedure, e.g., a series of actions to be executed, that is 
developed before actually carrying it out. This means that there is a time lag between planning and execution and consequences of actions will be difficult to foresee (delayed feedback). Third, design involves creating something that fulfils some need and satisfies some requirements. In experimental design, the main goal is to answer the research question and to discard alternative explanations for results found once the procedure is executed. These features constitute some of the sources of difficulty in experimental design processes.

In conclusion, experimental design is an important but neglected activity in labwork. The current article aims at finding ways of encouraging experimental design activities through the use of computer networks. Although the present work does not focus on direct learning gains, there are reasons to belief that design activities in themselves may contribute to learning (Hmelo et al. 2000). Design is thought to force students to think about structural, functional, and behavioural relationships between components of complex systems such as the human respiratory system. Similarly, experimental design in chemistry, besides its intrinsic value, could also deepen students understanding about chemical concepts, structures, functions and behaviours.

\section{Educaffix.net: a remote laboratory}

This remote laboratory is implemented to help students to design chemical experiments before performing them. Here, the expert chemical knowledge is first analyzed in order to implement the course content, a tutor and also to structure and organize the student activities. A concept mapping technique (Giordan et al. 1986, Novak 1988) is used to categorize, connect and display the relevant concepts in a comprehensive way.

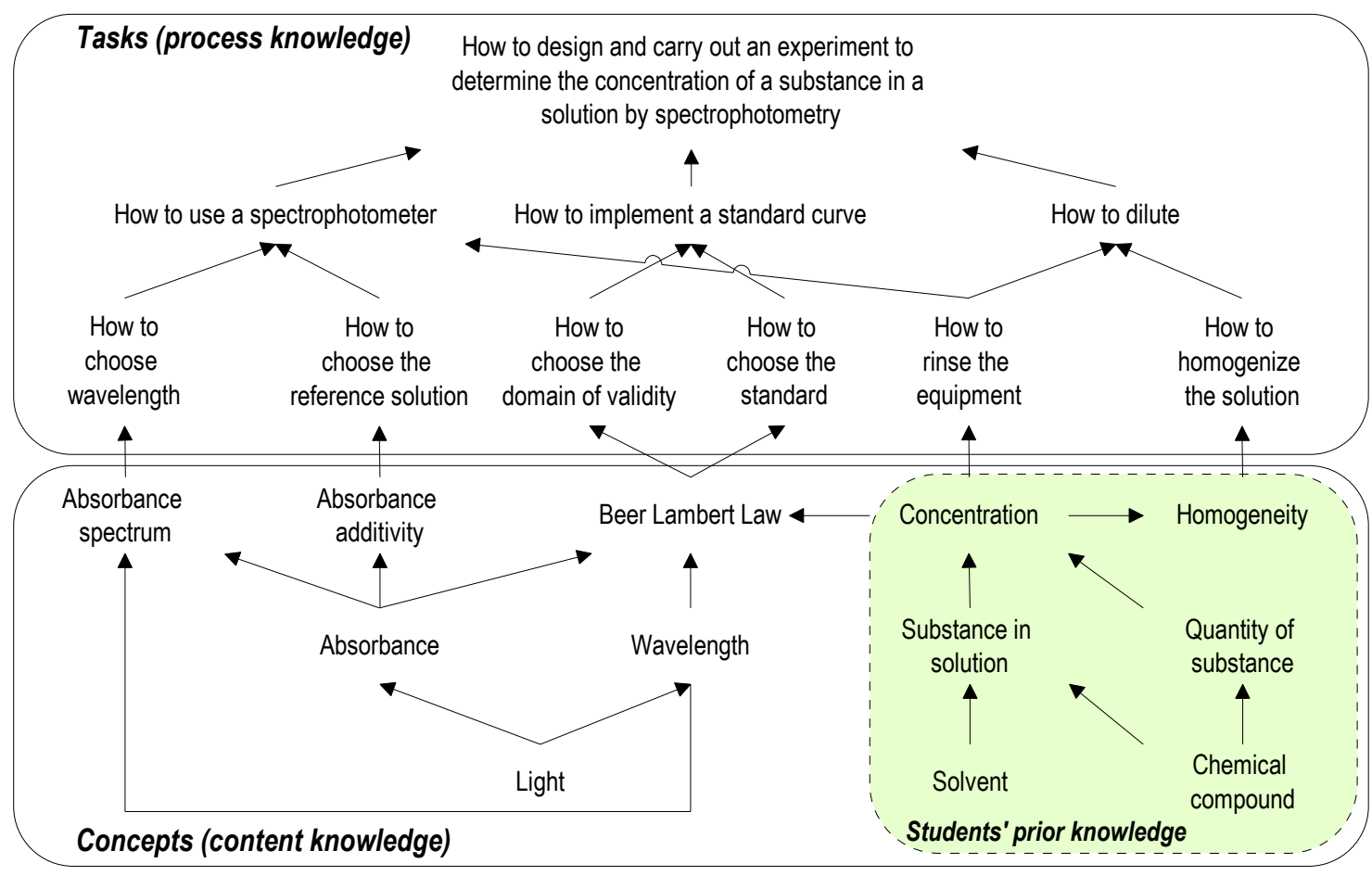

Figure 1. Relevant process and content knowledge. Arrows indicate that a task or concept is a prerequisite to another one, i.e. knowing about light is a prerequisite to knowing about wavelength. 


\section{Domain specific content}

It is very common in the field of chemistry or biochemistry to deal with a solution in which a compound needs to be quantified. For this purpose, several methods can be used, such as conductimetry, potentiometry, $\mathrm{pH}$-metry or spectrophotometry. These are all physical measurement methods that are appropriate in different situations. In Educaffix.net, students have to determine the concentration of the E124 dye in a solution of grenadine syrup by spectrophotometry. The chosen method, i.e. construction of a calibration curve, is widely used in chemistry. Learners have to resolve a technological problem, and in order to succeed, they need to mobilize chemistry knowledge to design the protocol, i.e. the procedure to be carried out in the specific context determined by the question. The French chemistry curriculum describes this kind of experiment for students enrolled in the last year of high school or in first year of college.

Figure 1 shows the tasks (upper part) and concepts (lower part) that play a role in the design and execution of an experiment to determine the concentration of a substance in a solution with a spectrophotometer. Each subtask is relevant for either one or both of the two main goals of experimental design: answer the question and control variance. For example, answering a research question about the determination of a concentration may involve the use of a spectrophotometer and a standard curve. Controlling variance involves optimally choosing the values of a number of parameters in the process (wavelength, reference solution, domain of validity, etc.). In addition, the tasks vary in the degree of requiring manual skill for their performance. For example, in order to obtain very precise measurements, the flasks need to be rinsed with the target solution. This subtask both needs to be specified in the experimental design (methodological component) and is essential once carrying out the experiment (manual component). Other manual components are present in the knowledge needed for manipulating the spectrophotometer, diluting a solution (changing its concentration) and homogenizing a solution. Although students need to acquire the knowledge concerning these tasks, the actual manual execution in Educaffix.net is taken over by the robot. The relation between tasks and concepts is as follows:

- Choose the wavelength for measurement by looking at the absorbance spectrum of the target chemical compound and taking the one that shows maximum absorbance of light.

- Choose the reference solution by using the additivity of absorbance spectra, i.e. taking the target solution minus the target compound.

- Choose the domain of validity according to Beer Lambert law, i.e. within the range of values for which there exists a linear relation between concentration and absorbance.

- Choose the standard, i.e. a solution with different concentrations of the target compound.

- Rinse the equipment with the target solution in order to avoid inadvertent changes in concentration prior to measurement.

- Homogenize the solution in order to avoid measurement errors due to concentration errors.

\section{Prestructuring into four stages and eight elementary steps}

In order to structure the task of the learner, the complete task of experimental design was divided into four main stages. These stages define the general method to be used: construction of a calibration curve in order to compare the results of the unknown sample to the standard. Other methods could have been chosen, such as the use of a unique standard solution to do the comparison or the direct 
determination of the E124 dye concentration by finding the value of its absorption coefficient in the literature. The four stages are the following:

- Selection of chemical compounds

- Preparation of a series of standard solutions

- Construction of the calibration curve

- Determination of the concentration of E124 in the grenadine syrup

To design the experimental procedure, the learner has to choose amongst eight elementary steps and place them in a correct order in the notebook. These steps correspond to technical procedures that will be executed by the robot (i.e. record a spectrum). In addition, the student has to think about, calculate and/or choose the values of corresponding parameters (i.e. choose the concentration of the different solutions used to implement the standard curve). Eight actions are proposed and listed below:

- Select a chemical compound

- Consult a data safety sheet

- Prepare a solution by dilution

- Set the baseline of the spectrophotometer

- Record a spectrum

- Record an absorption measure

- Rinse

- Homogenize

These actions were defined as general processes, independent of materials, and may be reused in other experimental situations. Each one is defined by a set of parameters that have to be specified by the learner before the action is recorded in the notebook. For example, when choosing the action "prepare a solution by dilution", the learner has to define the volume and the nature of the solution to be diluted, the total volume of the new solution and the nature of the solvent used to dilute. Specifying the parameters requires the process knowledge described above.

\section{The feedback of the artificial tutor}

Before the robot performs the experiment, the procedure is checked and validated by a tutor. This system automatically tests the validity of the procedure according to the criteria mentioned below: (i) will the proposed procedure allow an adequate answer to the research question? (ii) does the procedure allow sufficient control of sources of variance so that the results will be significant? It has to be noted that the learner has some latitude to choose the actions and their parameters: there is not a single procedure that will be positively recognized as the right solution by the artificial tutor. However, different procedures will produce results more or less easy to interpret.

The feedback of the artificial tutor is given on the student's request and is separated into three levels. It has the advantage to structure the answer a teacher would give. A general level shows the degree of completion for each of the four predefined stages and details can be obtained for each stage. The second level of feedback is the number and the type of errors. The third level is the detailed description of the error with direct access to the appropriate theory part in the textbook. The artificial tutor does not propose a correction of the experimental procedure but points out problems that then might be corrected by the learner. 


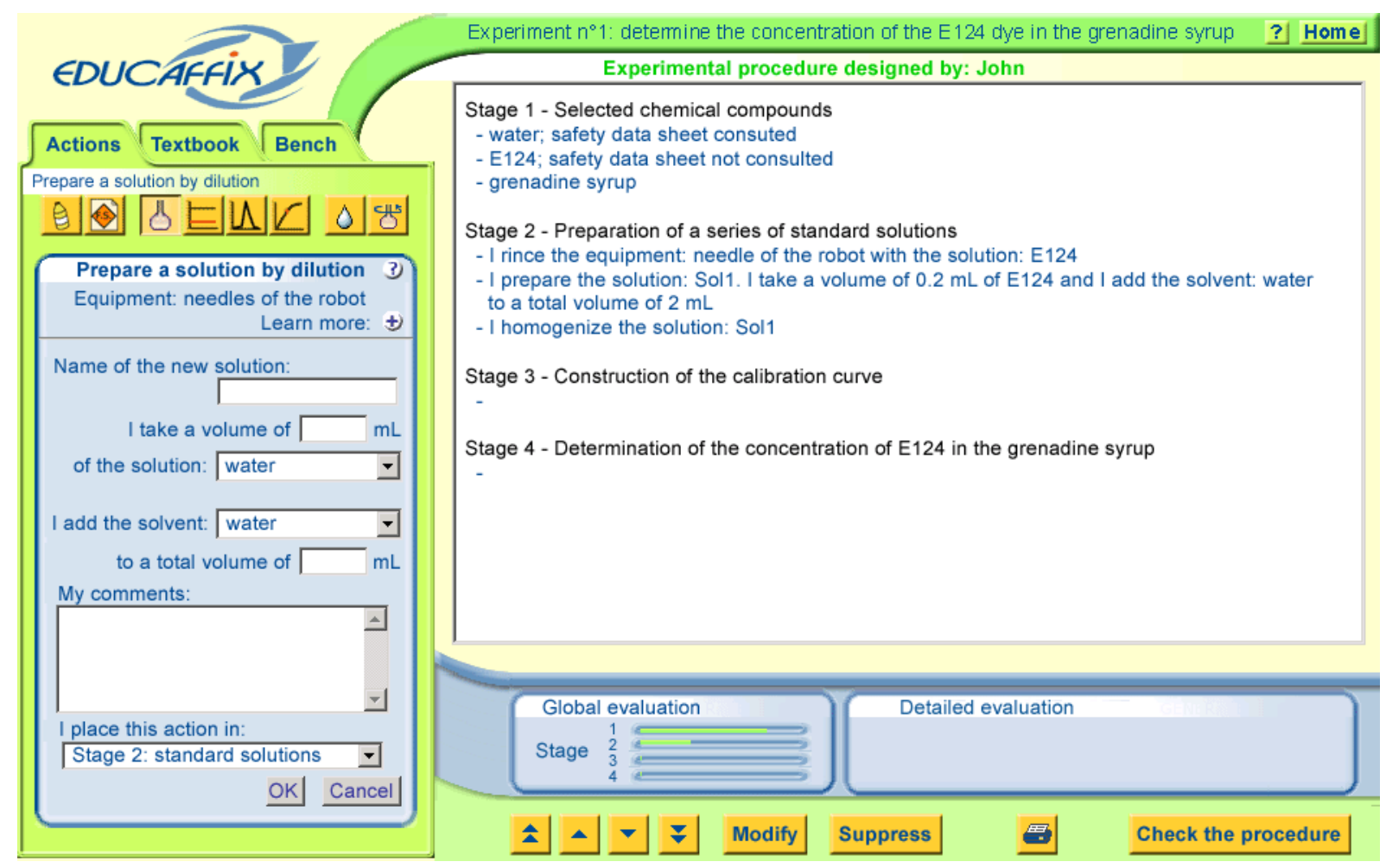

Figure 2. Interface of the Educaffix.net system

\section{The interface}

The Figure 2 shows an English translation of the Educaffix.net interface that is currently only available in French. This interface is provided to the student for designing the experimental procedure and watching the experiment run. It is divided into two parts. In the first part (on the right side) is the notebook, where the student-designed experimental procedure appears as a set of elementary steps categorized in the four pre-defined stages. Connected to the notebook are the buttons dedicated to the modification of its content: change the order of the elementary steps, modify or suppress an elementary step, print the experimental procedure or have it checked by the artificial tutor. When the latter button is used, a box dedicated to the artificial tutor's feedback appears below the notebook.

In the second part (on the left side of the interface), the learner can find the tools to build the experimental procedure and the panel to watch the experiment running. Three window tabs can be seen:

- Actions: elementary steps that can be chosen by clicking on one of the eight icons

- Textbook: documents containing domain knowledge

- Bench: allows watching the robot performing the actions once the experiment starts

\section{Experimental design in Educaffix.net}

In Educaffix.net a number of features of the setting naturally render experimental design more salient than other common objectives of labwork. These features are presented below. 


\section{Robot reduces importance of gestures}

A chemist makes gestures with arms and hands to operate devices and to handle fluids. However, it is not the handling of specific equipment, but the gathering of specific data that is at focus here. Therefore, the equipment used can as well be different from the one used in a regular laboratory. For example, in Educaffix.net, mixing of chemicals is done with a diluting robot and precise supplies of liquid are obtained with a syringe and needle instead of a graduated pipette as a human chemist would do.

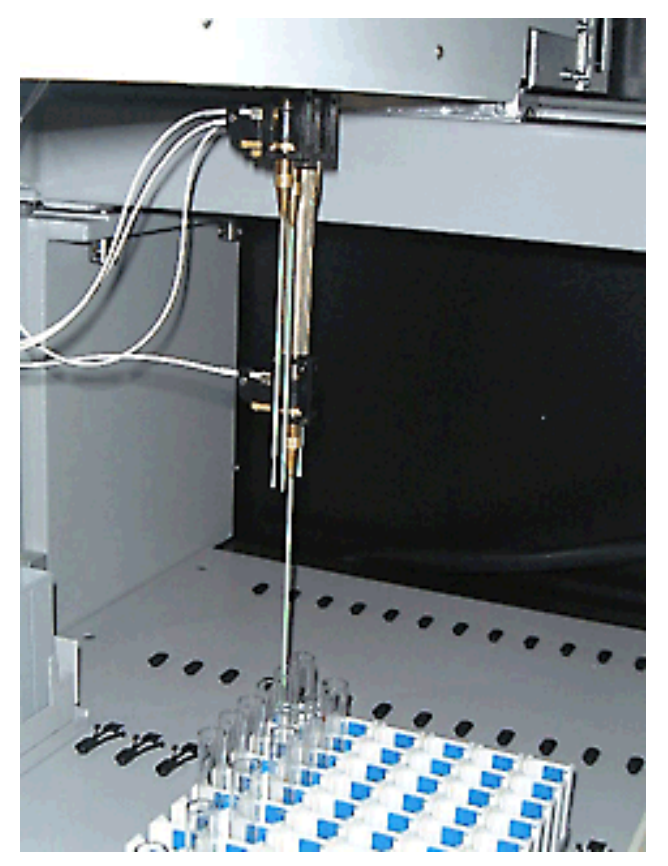

Figure 3. The needles of the robot

\section{Discrete steps replace continuous (perception-action) monitoring}

When preparing a solution by dilution, a human chemist puts a known amount of concentrated solution in a volumetric flask and then adds the right amount of solvent by watching graduation marks on the flask (and stops when the required volume is reached). Transmission times would in fact make this kind of perception-action monitoring very error prone because of delays between student action and the execution by the robot. Again, prevalence is given to the content validity (amounts of fluid), rather than resemblance to the real laboratory. The technical solution adopted in Educaffix.net involves adding a known volume of solvent to a concentrated solution instead of visually adjusting to the required final volume.

\section{Time sharing enforces serial task performance}

The robot can be used for one experiment at a time. Therefore, the equipment has to be time-shared by the students such that when a learner is connected to the remote laboratory, the equipment is not available for use by other students. This feature in fact does mirror the reality of experiments in laboratories involving expensive apparatus. The costs of carrying out an experiment on the basis of an incorrect or imprecise design are high. This feature of the setting naturally enforces the construction of a high quality experimental design before actually trying it out with the apparatus. 


\section{System performs security check}

Any experiment in chemistry involves security measures. Chemical compounds, even if they are not dangerous in terms of chemical reactions, can lead to problems. For example, water could damage electrical apparatus and cause explosion. It would not be feasible for a teacher to simultaneously attend to several students testing their experimental protocol. In Educaffix.net, a security check is performed and the system does not allow dangerous manipulations (cf. putting $5 \mathrm{~mL}$ of liquid in a 2 $\mathrm{mL}$ container).

These four features make the learning situation diverge from the real situation of expert chemist researchers. In other words, they reduce the fidelity of the learning situation (Alessi and Trolip 1991). However, the same features might have a beneficial effect on student activities and learning.

\section{Conclusions and future research}

This article presents a system for concentrating students' activities on experimental design as a prelaboratory preparation. The particular topic of spectrophotometry and how to determine the concentration of a compound in a solution was analysed in order to pay attention to technologyinduced changes when transposing expert knowledge and tasks to the learning situation. The resulting system, Educaffix.net, is a remote laboratory in which learners have to design an experiment, with the help of the artificial tutor, before it is carried out by the distant robot.

The design process is the result of a balance between theoretical conjectures about learning with ICT and technological opportunities of ICT. The main pedagogical aims were maintained throughout this process: students have to answer a question, construct the experimental procedure themselves, look up theoretical references online, and check their procedures by soliciting the artificial tutor. Likewise, the study of actual student activities in the created situation will have to deal with theoretical notions about learning with technology and the influences of technology. Such a study into the use of Educaffix.net might reveal new issues to be considered in developing learning environments for labwork and allow comparisons with other forms of student activity. Rollnick et al. (2001) for example worked on the effectiveness of adequate student preparation before labwork. They asked students to write a synopsis of an experiment: this implies an appreciation of the theory underlying the experiment and a general view of the procedure to be used. However, this procedure also was shown to pose problems.

The use of Educaffix.net will be studied with about a hundred students in high school from the south east of France. Our aim is to study how the students design their experiment. The hypotheses are (i) that Educaffix.net will help students to design an experiment linked to the specific problem at hand; (ii) that the task of experimental design will help students to understand the relations between experimental procedures and the domain processes and knowledge necessary to solve the problem; (iii) and that students misconceptions will induce difficulties and errors in the activity of designing the experiment. The results of this study might inform theories of learning with technology as well as the design of future learning situations. 


\section{Acknowledgements}

The authors thank the French ministry of Education and Research for financial support, and the EDUCAFFIX company for the development of the software and the robot interfacing.

\section{References}

Alessi, S. M. \& Trolip, S. R. (1991). Computer-based instruction: methods and developments development (2nd edition). Englewood Cliffs, NJ: Prentice Hall.

Arce, J. \& Betancourt, R. (1997). Student-designed experiments in scientific lab instruction. Journal of College Science Teaching, 27, 114-118.

Balacheff, N. (1996). Advanced educational technology: knowledge revisited. In T. T. Liao (Ed.), Advanced Educational Technology: Research Issues and Future Potential (NATO ASI Series F: Computer and Systems Sciences) (pp. 1-20). Berlin: Springer

Bécu-Robinault, K. (1997). Rôle de l'expérience en classe de physique dans l'acquisition des connaissances sur les phénomènes énergétiques [The role of experiments in physics teaching in acquiring knowledge about energy phenomena]. Unpublished doctoral dissertation, Université Claude Bernard, Lyon, France.

Chevallard, Y. (1985), La transposition didactique [Didactical transposition]. Grenoble: la pensée sauvage.

Cooper, M., Donnelly, A. \& Ferreira, J. (2002). Remote controlled experiments for teaching over the internet: a comparison of approaches developed in the pearl project. Paper presented at the ASCILITE 2002 Conference, December 8-11, 2002, Auckland, New Zealand.

Darley, B. (1996). Exemple d'une transposition didactique de la démarche scientifique dans un TP de biologie en DEUG 2ème année [Example of a didactical transposition of a scientific approach in labwork in biology in the second year of university]. Didaskalia, 9, 31-56.

De Jong, T. \& van Joolingen, W. R. (1998). Scientific discovery learning with computer simulations of conceptual domains. Review of Educational Research, 68, 179-202.

De Vries, E. (1994). Structuring information for design problem solving. Unpublished doctoral dissertation, Eindhoven University of Technology, Eindhoven, The Netherlands.

De Vries, E. (2003). Educational technology and multimedia from a cognitive perspective: Knowledge from inside the computer, onto the screen, and into our heads? In H. van Oostendorp (Ed.), Cognition in a digital world (pp. 155-174). Mahwah, NJ: Lawrence Erlbaum Associates.

Desautel, J., Larochelle, M., Gagné, B. \& Ruel, F. (1993). La formation à l'enseignement des sciences : le virage épistémologique [Teacher training in science: the epistemological turn]. Didaskalia, 1, 49-67.

Giordan, A., Pochon, J., Aimar, C., Host, V., Le Jan, E., Stern, G., Vilain, C., Catesson, A.-M., Grosbois, M., Rieutord, M., Taugourdeau, J. \& Bosch-Vidal, C. (1986) Preliminary analysis to build an integrative conceptual network for biological education at university level. European Journal of Science Education, 8, 251-261.

Hmelo, C. E., Holton, D. H. \& Kolodner, J. L. (2000) Designing to learn about complex systems. The Journal of the Learning Sciences, 9, 247-298.

Kozma, R., Chin, E., Russel, J. \& Marx, N. (2000). The roles of representations and tools in the chemistry laboratory and their implications for chemistry learning. The Journal of the Learning Sciences, 9, 105-143. 
Novak, J. D. (1988). Learning science and the science of learning. Studies in Science Education, 15, 77-101.

Orlandi, E. (1991). Conceptions des enseignants sur la démarche expérimentale [Teacher conceptions on the experimental approach], Aster, 13, 111-132.

Rollnick, M., Zwane, S., Staskun, M., Lotz, S. \& Green, G. (2001). Improving pre-laboratory preparation of first year university chemistry students. International Journal of Science Education, 23, 1053-1071.

Schraagen, J. M. (1993). How experts solve a novel problem in experimental design. Cognitive Science, 17, 285-309.

Senese, F.A., Bender, C. \& Kile, J. (2000). The Internet chemistry set: web-based remote laboratories for distance education in chemistry. Interactive Multimedia Electronic Journal of ComputerEnhanced Learning, 2, $\mathrm{n}^{\circ} 2$.

Séré, M.-G. (2002). Towards renewed research questions from the outcomes of the European project labwork in science education. Science Education, 86, 624-644.

Séré, M.-G. \& Beney, M. (1997). Le fonctionnement intellectuel d'étudiants réalisant des expériences : observation de séances de travaux pratiques en premier cycle universitaire scientifique [Students thinking while doing experiments: observation of labwork in the first years of higher education]. Didaskalia, 11, 75-102.

Tiberghien, A., Veillard, L., Le Maréchal, J.-F., Buty, C. \& Millar, R. (2001). An analysis of labwork tasks used in science teaching at upper secondary school and university levels in several European countries. Science Education, 85, 483-508.

Trgalová, J. (2003). Systèmes de formation à distance. Proposition d'une typologie [Distance learning systems, a typology]. In C. Desmoulins, P. Marquet, \& D. Bouhineau (Eds.), EIAH2003 Environnements Informatiques pour l'Apprentissage Humain. Proceedings of EIAH 2003, April 15-17, Strasbourg, France (pp. 563-566). Paris: INRP. 